\title{
A FURTHER REFINEMENT FOR COEFFICIENT ESTIMATES OF UNIVALENT FUNCTIONS
}

\author{
DAVID HOROWITZ
}

\begin{abstract}
The coefficient inequalities of FitzGerald are used to show that if $f(z)=z+a_{2} z^{2}+a_{3} z^{3}+\ldots$ is analytic and univalent in the unit disc, then $\left|a_{n}\right|<(1.0657) n$. The technique used to obtain this bound cannot yield a result better than $\left|a_{n}\right|<(1.0599) n$.
\end{abstract}

Let

$$
S=\left\{f(z)=z+\sum_{n=2}^{\infty} a_{n} z^{n}: f \text { is analytic and univalent in the unit disc }\right\} .
$$

The Bieberbach Conjecture asserts that if $f(z)=z+a_{2} z^{2}+a_{3} z^{3}+\ldots$ is in $S$, then $\left|a_{n}\right| \leqslant n(n=2,3,4, \ldots)$. This conjecture is the most renowned in geometric function theory, and much research and investigation has been devoted to establishing its validity. It has long been known [10] that the Bieberbach Conjecture is of the correct order in $n$, i.e. that there is some $C<\infty$ such that $\left|a_{n}\right|<C n(n=2,3,4, \ldots)$. Table 1 outlines the history of successive attempts to improve the number $C$.

\begin{tabular}{|c|c|c|c|}
\hline Name & Year & $\left|a_{n}\right|<C n$ & \\
\hline Littlewood [10] & 1923 & $\left|a_{n}\right|<e n$ & $\simeq 2.7183 n$ \\
\hline Landau [7] & 1929 & $\left|a_{n}\right|<\left(\frac{1}{2}+\frac{1}{\pi}\right) e n$ & $\simeq 2.2244 n$ \\
\hline Goluzin [4] & 1946 & $\left|a_{n}\right|<\frac{3}{4}$ en & $\simeq 2.0388 n$ \\
\hline Bazilevič [1] & 1947 & $\left|a_{n}\right|<\frac{9}{4}\left(\frac{1}{\pi} \int_{0}^{\pi} \frac{\sin x d x}{x}+.2649\right) n$ & $\simeq 1.9240 n$ \\
\hline Milin [8], [9] & 1949 & $\left|a_{n}\right|<\frac{1}{2}$ en +1.80 & $\begin{array}{r}\simeq 1.3592 n \\
+1.80\end{array}$ \\
\hline Bazilevič [2] & 1949 & $\left|a_{n}\right|<\frac{1}{2} e n+1.51$ & $\begin{array}{r}\simeq 1.3592 n \\
+1.51\end{array}$ \\
\hline Milin [11] & 1964 & $\left|a_{n}\right|<\frac{(e-1)}{1.6} n$ & $\approx 1.2427 n$ \\
\hline FitzGerald [3] & 1971 & $\left|a_{n}\right|<\left(\frac{7}{6}\right)^{1 / 2} n$ & $\simeq 1.0802 n$ \\
\hline Horowitz [6] & 1975 & $\left|a_{n}\right|<\left(\frac{209}{140}\right)^{1 / 6} n$ & $\simeq 1.0691 n$ \\
\hline
\end{tabular}

\section{TABLE 1}

Received by the editors June 29, 1977.

AMS (MOS) subject classifications (1970). Primary 30A34. 
FitzGerald [3] has suggested the strongest method to date for improving the number $C$. Subsequently this author [6] applied FitzGerald's technique to improve the coefficient estimate. In this paper, FitzGerald's method is refined once again, and the resulting improvement is stated in the following

TheOREM. If $f(z)=z+a_{2} z^{2}+a_{3} z^{3}+\ldots$ is in $S$, then

$$
\left|a_{n}\right|<\left(\frac{1,659,164,137}{681,080,400}\right)^{1 / 14} n<1.0657 n \quad(n=2,3,4, \ldots) .
$$

The proof of the theorem is based upon the following reformulation of FitzGerald's Coefficient Inequality [3].

Lemma. If $f(z)=z+a_{2} z^{2}+a_{3} z^{3}+\ldots$ is in $S, \lambda_{1}, \lambda_{2}, \ldots \lambda_{L}$ are complex numbers, and $n_{1} \leqslant n_{2} \leqslant \cdots \leqslant n_{L}$ are positive integers, then

$$
\begin{aligned}
& \left.\left.\left|\sum_{k=1}^{L} \lambda_{k}\right| a_{k}\right|^{2}\right|^{2} \\
& \qquad \sum_{k=1}^{2 L}\left|a_{k}\right|^{2} \sum_{j=[(k+1) / 2]}^{L} \sum_{l=|k-j|}^{j} \beta(j, l)(l-|j-k|) \operatorname{Re}\left(\lambda_{j} \bar{\lambda}_{l}\right)
\end{aligned}
$$

where

$$
\beta(j, l)= \begin{cases}1 & \text { if } j=l, \\ 2 & \text { if } j \neq l\end{cases}
$$

and $[x]$ denotes the greatest integer $\leqslant x$ by standard notation.

Inequality (2) differs from FitzGerald's original inequality in that (i) the order of summation on the right-hand side has been changed, and (ii) it has been generalized to include complex coefficients $\left\{\lambda_{j}\right\}$. The proof of this lemma involves a straightforward counting argument [5] and is omitted here.

Proof of TheORem. In (2) set $L=n, \lambda_{1}=\lambda_{2}=\cdots=\lambda_{n-1}=0$, and $\lambda_{n}=1$ to obtain

$$
\left|a_{n}\right|^{4} \leqslant \sum_{k=1}^{2 n} \Lambda_{1}(n, k)\left|a_{k}\right|^{2}
$$

where

$$
\Lambda_{1}(n, k)=n-|n-k| \quad(k=1,2,3, \ldots, 2 n) .
$$

Next in (2) let $L=2 n$ and $\lambda_{k}=\Lambda_{1}(n, k)$ for $k=1,2,3, \ldots, 2 n$. Then the left-hand side of (2) will have the form of the right-hand side of (3), and therefore it follows that

$$
\left|a_{n}\right|^{8} \leqslant \sum_{k=1}^{4 n} \Lambda_{2}(n, k)\left|a_{k}\right|^{2}
$$

where

$$
\begin{array}{r}
\Lambda_{2}(n, k)=\sum_{j=[(k+1) / 2]}^{2 n} \sum_{l=|k-j|}^{j} \beta(j, l)(l-|j-k|) \Lambda_{1}(n, j) \Lambda_{1}(n, l) \\
(k=1,2,3, \ldots, 4 n) .
\end{array}
$$


Now in (2) let $L=4 n$ and $\lambda_{k}=\Lambda_{2}(n, k)$ for $k=1,2,3, \ldots, 4 n$. Then the left-hand side of (2) will have the form of the right-hand side of (5), and therefore it follows that

$$
\left|a_{n}\right|^{16} \leqslant \sum_{k=1}^{8 n} \Lambda_{3}(n, k)\left|a_{k}\right|^{2}
$$

where

$$
\begin{array}{r}
\Lambda_{3}(n, k)=\sum_{j=[(k+1) / 2]}^{4 n} \sum_{l=|k-j|}^{j} \beta(j, l)(l-|j-k|) \Lambda_{2}(n, j) \Lambda_{2}(n, l) \\
(k=1,2,3, \ldots, 8 n) .
\end{array}
$$

In fact it is easy to continue this process and establish for any positive integer $m$

$$
\left|a_{n}\right|^{2^{m+1}} \leqslant \sum_{k=1}^{2^{m} n} \Lambda_{m}(n, k)\left|a_{k}\right|^{2}
$$

where $\Lambda_{m}(n, k)$ is defined recursively by

$$
\begin{aligned}
& \Lambda_{1}(n, k)= n-|n-k| \quad(k=1,2,3, \ldots, 2 n) \\
& \Lambda_{r}(n, k)=\sum_{j=[(k+1) / 2]}^{2^{m-1} n} \sum_{l=|k-j|}^{j} \beta(j, l)(l-|j-k|) \Lambda_{r-1}(n, j) \cdot \Lambda_{r-1}(n, l) \\
&\left(k=1,2,3, \ldots, 2^{m} n ; r=2,3, \ldots,\right) .
\end{aligned}
$$

To establish (1) suppose that

$$
C=\sup _{n} \sup _{f \in S}\left\{\frac{\left|a_{n}\right|}{n}\right\} .
$$

If $\varepsilon>0$, then there is a positive integer $n$ and a function $f(z)=z+a_{2} z^{2}+$ $a_{3} z^{3}+\ldots$ in $S$ such that

$$
n(C-\varepsilon)<\left|a_{n}\right| \cdot
$$

From (7), (11), and (12) it follows that

$$
n^{16}(C-\varepsilon)^{16} \leqslant C^{2} \sum_{k=1}^{8 n} \Lambda_{3}(n, k) k^{2} .
$$

The sum in the right-hand side of (13) is too lengthy to be calculated by hand, but it only involves sums of the form $\sum_{k=1}^{s} k^{t}$ where $s$ and $t$ are positive integers. All of these sums are polynomials in $s$ and $t$ with rational coefficients. Thus closer examination discloses that the sum on the right-hand side of (13) is a polynomial $P(n)$ of degree 16 with rational coefficients. Moreover whenever $n$ is a positive integer, then so is $P(n)$. A computer program was written to evaluate $P(n)$ for 17 different positive integers, and the resulting values were combined with the Lagrange Interpolation Formula [12] to derive that 


$$
\begin{aligned}
P(n)= & \frac{1,659,164,137}{681,080,400} n^{16} \\
& -\frac{24,078,479}{14,968,800} n^{14}+\frac{5,621,807}{14,968,800} n^{12} \\
& -\frac{2,102,099}{9,525,600} n^{10}+\frac{94,789}{1,360,800} n^{8} \\
& -\frac{200,887}{7,484,400} n^{6}+\frac{87,797}{6,810,804} n^{4} \\
& -\frac{128}{10,395} n^{2} .
\end{aligned}
$$

By examining the terms in (14) pairwise it can be shown that

$$
P(n) \leqslant \frac{1,659,164,137}{681,080,400} n^{16} \text { for } n \geqslant 2 \text {. }
$$

Since $\varepsilon>0$ was chosen arbitrarily, it follows from (13) and (15) that

$$
C^{14} \leqslant \frac{1,659,164,137}{681,080,400}
$$

whence

$$
C \leqslant\left(\frac{1,659,164,137}{681,080,400}\right)^{1 / 14}<1.0657 .
$$

This proves (1).

It should be noted that the estimate (1) can continually be improved by appealing to (9) with ever-increasing values of $m$. However the number of calculations becomes prohibitive while the refinements become minuscule. For example if $m=4$ and $n=16$, then (9) becomes

$$
\left|a_{16}\right|^{32} \leqslant \sum_{k=1}^{256} \Lambda_{4}(16, k)\left|a_{k}\right|^{2} .
$$

Any estimate that can be obtained on the right-hand side of (16) cannot be better than that which would follow by replacing $\left|a_{k}\right|$ by $k$ in this expression, since the Koebe function

$$
f(z)=\frac{z}{(1-z)^{2}}=\sum_{k=1}^{\infty} k z^{k}
$$

is in $S$. By doing so, the right-hand side of (16) sums to

$$
2191891460910315201087792918069979806720>(16)^{32}(1.0599)^{32}
$$

from which it follows that the improved estimate described above can be no better than

$$
\left|a_{n}\right|<1.0599 n \text {. }
$$

Thus while the Bieberbach Conjecture might still be a consequence of (2), any subsequent attempts to establish it by solely using the above iterative technique will be fruitless.

The author would like to thank H. J. Samet of the Department of 
Computer Science, University of Maryland for programming some of the lengthy calculations contained herein, and C. H. FitzGerald of the Department of Mathematics, University of California, San Diego for his insights and suggestions.

\section{REFERENCES}

1. I. E. Bazilevic, Improvement of estimates for the coefficients of univalent functions, Mat. Sb. 22 (64) (1948), 381-390. (Russian) MR 10, 186.

2. On distortion theorems and coefficients of univialent functions, Mat. Sb. 28 (70) (1951), 147-164. (Russian) MR 12, 600.

3. C. H. FitzGerald, Quadratic inequalities and coefficient estimates for schlict functions, Arch. Rational Mech. Anal. 46 (1972), 356-368. MR 49 \# 557.

4. G. M. Goluzin, On the coefficients of univalent functions, Mat. Sb. 22 (64) (1948), 373-380. (Russian) MR 10, 186.

5. D. A. Horowitz, Applications of quadratic inequalities in the theory of univalent functions, Doctoral dissertation, University of California, San Diego, 1974.

6. A refinement for coefficient estimates of univalent functions, Proc. Amer. Math. Soc. 54 (1976), 176-178. MR 53 \#792.

7. E. Landau, Uber schlichte Funktionen, Math. Z. 30 (1929), 635-638. FM 55, 187.

8. N. A. Lebedev and I. M. Milin, On the coefficients of certain classes of analytic functions, Dokl. Akad. Nauk SSSR 67 (1949), 221-223. (Russian) MR 11, 339.

9. On the coefficients of certain classes of analytic functions, Mat. Sb. 28 (70) (1951), 359-400. (Russian) MR 13, 640.

10. J. E. Littlewood, On inequalities in the theory of functions, Proc. London Math. Soc. (2) 23 (1925), 481-519.

11. I. M. Milin, Estimation of coefficients of univalent functions, Dokl. Akad. Nauk SSSR 160 (1965), 769-771 = Soviet Math. Dokl. 6 (1965), 196-198. MR 30 \# 3206.

12. J. L. Walsh, Interpolation and approximation by rational functions in the complex domain, Amer. Math. Soc. Colloq. Publ., vol. 20, Amer. Math. Soc., Providence, R. I., 1969.

Department of Mathematics, Golden West College, Huntington Beach, California 92647 